Check for updates

Cite this: RSC Adv., 2018, 8, 31071

\title{
Expression of Ac-PK2 protein from AcMNPV improved the progeny virus production via regulation of energy metabolism and protein synthesis $\uparrow$
}

\begin{abstract}
Lili Wei, Aihua Liang and Yuejun Fu ID *
Baculovirus encoded PK2 protein can increase viral fitness through inhibition of the elF2 $\alpha$ family kinases activity. Previous studies indicated that the virus might take over the control of cellular machinery postinfection, which would impose a high metabolic burden to infected insect cells. Here we showed that elF $2 \alpha$ phosphorylation decreased, with concomitant up-regulation of total and heterologous protein synthesis in AcMNPV-PK2-EGFP infected Sf9 cells and the larvae of Spodoptera exigua. Simultaneously, the lactic acid accumulation decreased and the uptake of glucose increased in AcMNPV-PK2-EGFP infected Sf9 cells. We proposed a model that Ac-PK2 protein overexpression would help protein synthesis by inhibiting elF2 $\alpha$ phosphorylation, which provided a more favorable scenario to support the efficient replication of the virus by re-directing the cellular metabolism toward ATP production. Finally, we confirmed that ACMNPV-PK2-EGFP could improve the production of progeny virus in infected Sf9 cells and enhance insecticidal activity against Spodoptera exigua larvae.
\end{abstract}

Received 16th June 2018

Accepted 26th August 2018

DOI: 10.1039/c8ra05172k

rsc.li/rsc-advances

highlighted that baculovirus could redirect energy fluxome

\section{Introduction}

Autographa californica multiple nucleopolyhedrosis virus (AcMNPV) is a kind of insect baculovirus, whose genome contains about 154 ORFs. ${ }^{1}$ AcMNPV specifically infects lepidopteran insects and has been extensively used as a biological insecticide and eukaryotic expressing system for exogenous gene expression.

Ac-pk2 gene is the ORF123 of the AcMNPV genome and encodes 215 amino acids. Ac-PK2 contains six signature motifs of serine- and threonine-specific protein kinases. Generally, AcPK2 protein doesn't have kinase function since it lacks catalytic subdomains. ${ }^{2}$ Translation initiation factor $2 \alpha$ family kinases can phosphorylate eukaryotic eIF $2 \alpha$ and suppress protein translation initiation once cells are suffering from certain pressure conditions. ${ }^{3}$ Ac-PK2 protein performs its function by combining with the eIF $2 \alpha$ kinase $\mathrm{N}$-domain to form a heterogeneous dimer to inhibit its activity. Because the sequence is highly homologous, we speculate that AcMNPV obtains the $p k 2$ gene by horizontal gene transfer from the host. ${ }^{4}$

Baculovirus infection increases the metabolic stress of the host cell. ${ }^{5} \mathrm{Sf} 9$ cells can make better use of glucose to provide energy by the TCA cycle. ${ }^{6}$ The report from Monteiro et al.

Key Laboratory of Chemical Biology and Molecular Engineering of Ministry of Education, Institute of Biotechnology, Shanxi University, Taiyuan 030006, China. E-mail: yjfu@sxu.edu.cn; Fax: +86 351 7011499; Tel: +86 3517016125

$\dagger$ Electronic supplementary information (ESI) available. See DOI: 10.1039/c8ra05172k toward ATP production and provide a suitable environment for generation of progeny virus during the infection process. They also found that the by-product accumulation in host cell reduced after infection. ${ }^{7}$

Spodoptera exigua is an important injurious insect to agricultural production. It has developed a great resistance and multiple resistance to a lot of pesticides. ${ }^{8}$ Therefore, research and development of biological insecticide is at all imminent. In our previous work, we constructed recombinant virus AcMNPV$B m \mathrm{~K}$ IT, and found that it obviously improved the anti-insect activity of baculovirus. ${ }^{9}$

In the present work, AcMNPV-PK2-EGFP was constructed by Bac-to-Bac system to study the fitness and insecticidal activity of AcMNPV after the overexpression of Ac-PK2 protein. Our results showed that Ac-PK2 protein could inhibit eIF2 $\alpha$ kinase phosphorylation in Sf9 cell, compared with AcMNPV treatment group. To detect the effect of Ac-PK2 on host cell metabolism, we examined protein synthesis, glucose consumption, lactic acid accumulation, ATP content and hexokinase activity in AcMNPV-PK2-EGFP infected Sf9 cells. We speculated that overexpression of Ac-PK2 protein mediated by AcMNPV improved the energy metabolism of host cells to help the replication of progeny virus. Insect feeding bioassay was performed to provide a more thorough discussion of the insectresistant efficiency of AcMNPV, AcMNPV-PK2-EGFP, AcMNPV$B m \mathrm{~K}$ IT and AcMNPV-PK2-EGFP + AcMNPV-BmK IT against Spodoptera exigua larvae. 


\section{Materials and methods}

\section{Cell, insects, virus}

We cultured Sf9 cells using SIM SF serum free medium $\left(27^{\circ} \mathrm{C}\right)$. We purchased Spodoptera exigua eggs from Baiyun biotechnology Corp Ltd. AcMNPV was maintained in our laboratory, and recombinant AcMNPV-BmK IT constructed in our lab previously. ${ }^{9}$

\section{Construction of recombinant viruses}

For recombinant virus AcMNPV-PK2-EGFP or AcMNPV-RenillaRFP, the transcription of foreign $p k 2$ was cloned into pFastBacDual-egfp, then transformed into DH10B cells to get bacmidpk2-egfp. The construction process of bacmid-renilla-rfp was analogous.

For transfection, Sf9 cells were transfected with Bacmid-pk2egfp or Bacmid-renilla-rfp using FuGENE 6 Transfection reagent (Promega, USA). After three days, green or red fluorescent protein (EGFP/RFP) expression in transfected Sf9 cells was examined using fluorescence microscopy.

\section{Plaque assay}

AcMNPV or AcMNPV-PK2-EGFP infected Sf9 cells at 5 MOI. A small amount of supernatant was harvested at 24,48 and $72 \mathrm{~h}$, then $\mathrm{BV}$ production was determined by plaque assay. ${ }^{10}$ In brief, Sf9 cells were cultured in 6-well plates, and $500 \mu \mathrm{l}$ serially diluted AcMNPV or AcMNPV-PK2-EGFP virus suspension was added. Then, cells were standing for $1 \mathrm{~h}$ at $27{ }^{\circ} \mathrm{C}$, and were washed using PBS before nutrient agarose was added. Plaques were examined 5 days later. ${ }^{11}$

\section{RT-PCR analysis}

Total RNA were extracted from AcMNPV or AcMNPV-PK2-EGFP treated Sf9 cells. Then, cDNA was obtained by reverse transcription. Q-PCR was performed using StepOne ${ }^{\mathrm{TM}}$ Real-Time PCR System (Applied Biosystem). The sequence of primers (pk2 and $\beta$-actin) was showed in Table S1. $\dagger$ We chose $\beta$-actin gene as a reference gene, and we calculated relative transcription level by the $2^{-\Delta \Delta C_{\mathrm{T}}}$ method.

\section{Western blot analysis}

We firstly determined protein concentration by BCA Protein Assay Kit (Solarbio biological co., LTD, China). Then the protein samples were separated using 10\% SDS-PAGE, and target bands were transferred to PVDF membranes, then blocked in blocking buffer for $1 \mathrm{~h}$. Primary antibody was incubated overnight at $4{ }^{\circ} \mathrm{C}$, then washed with PBST 15 minutes per each rinse stage, and triple rinse was performed. Rabbit specific fluorescent secondary antibody (IRDye 800CW, diluted at $1: 20$ 000, Licor, USA) was incubated for $1 \mathrm{~h}$ at room temperature, then washed with PBST 15 minutes per each rinse stage, and triple rinse was performed. ODYSSY CLx (Gene Company Limited, USA) was used to detected the target band.

\section{Renilla luciferase activity test}

In order to detect the influence of AcMNPV-PK2-EGFP on protein translation, Sf9 cells were co-infected with AcMNPV-PK2-EGFP/ AcMNPV-Renilla-RFP or AcMNPV/AcMNPV-Renilla-RFP. Renilla luciferase activity was determined using the Renilla-Glo ${ }^{\circledR}$ Luciferase Assay System (Promega) and GloMax ${ }^{\mathrm{TM}}$ 20/20 follow the instruction. Renilla luciferase activity in each treatment group was compared to indicate the change fold at $12 \mathrm{~h}$.

\section{Determination of glucose, lactic acid, ATP content and HK activities}

The culture supernatant in AcMNPV or AcMNPV-PK2-EGFP treatment group was collected to detect glucose consumption (Shanghai rongsheng pharmaceutical co., LTD) and lactic acid (Nanjing Jiancheng co., LTD), and cells were harvested to detect the ATP content and HK activity (Nanjing Jiancheng co., LTD) at 12-72 h p.i. Experiment operation was following the manufacturer's manual. Protein concentration was detected by Bicinchoninic Acid (BCA) Protein Assay (Solarbio biological co., LTD).

\section{Flow cytometry analysis of cell apoptosis}

Sf9 cells in AcMNPV-PK2-EGFP or AcMNPV treatment group were harvested at 24, 48 and $72 \mathrm{~h}$. Then, PI and annexin-V-APC (KeyGEN BioTECH Co., China) were added to stain cells (KeyGEN BioTECH Co., China). After 20 min incubating at room temperature without exposure to light, cells were analyzed on a FACSCalibur (BD Biosciences Company, USA), and data analysis was performed using Cell Quest software (Becton Dickinson). A total of $5 \times 10^{5}$ events were counted for each sample.

\section{Anti-insect bioassay}

In this study, we chose Spodoptera exigua larvae to analyze the virulence of AcMNPV, AcMNPV-PK2-EGFP, AcMNPV-BmK IT, and AcMNPV-PK2-EGFP + AcMNPV-BmK IT. The second instar larvae were reared in 12-well microtiter plates under the condition of $29 \pm 2{ }^{\circ} \mathrm{C}$ with a piece of artificial diet $\left(27 \mathrm{~mm}^{3}\right)$ added $20 \mu \mathrm{l}$ of each viruses $\left(1 \times 10^{7} \mathrm{pfu} \mathrm{ml}^{-1}\right)$ daily for five days, respectively. The weight of larvae, fatality rate, pupation rate and eclosion rate of every treatment group were counted daily to evaluate the insect-resistant activity of viruses.

\section{Results}

\section{Construction of recombinant virus}

The Ac-pk2 gene in pFastBauDual-pk2-egfp, and the renilla gene in pFastBauDual-renilla-rfp were obtained by polymerase chain reaction. DNA sequencing confirmed that pFastBauDual-pk2egfp and pFastBauDual-renilla-rfp were constructed successfully (Fig. 1a and c). The recombinant Bacmid-pk2-egfp and Bacmidrenilla-rfp were identified by PCR, then the Bacmid-pk2-egfp or Bacmid-renilla-rfp was transfected into Sf9 cells, respectively. Cells were observed for EGFP/RFP using fluorescence microscopy at $120 \mathrm{~h}$ post-transfection. Then, culture medium of transfectedcells was collected to get recombinant virus AcMNPV-PK2-EGFP or AcMNPV-Renilla-RFP (Fig. 1b and d). 
AcMNPV-PK2-EGFP improved the expression of pk2 gene, and decreased eIF $2 \alpha$ phosphorylation

We detected the transcriptional expression profile of $p k 2$ gene in infected cells. The expression of $p k 2$ in AcMNPV infected Sf9 cells increased from 12 to $36 \mathrm{~h}$ p.i., then reduced at 36-72 h p.i. (Fig. S1a $\dagger$ ). In AcMNPV-PK2-EGFP infected Sf9 cells, the mRNA level of Ac-pk2 was 1.65-fold, 2.58-fold, 2.67-fold, 2.90-fold higher than that in AcMNPV infected Sf9 cell at 36, 48, 60, $72 \mathrm{~h}$ p.i., respectively (Fig. $\mathrm{S} 1 \mathrm{~b} \dagger$ ). These results indicated that the recombinant virus AcMNPV-PK2-EGFP could significantly increase the transcription level of $p k 2$.

To determine whether AcMNPV-PK2-EGFP can reduce eIF2 $\alpha$ phosphorylation, we collected AcMNPV or AcMNPV-PK2-EGFP infected Sf9 cells to perform western blot. Fig. 2a showed that PK2-EGFP fusion protein was firstly observed at $24 \mathrm{~h}$ p.i., then increased continuously until $72 \mathrm{~h}$ p.i. Phosphorylated eIF2 $\alpha$ in AcMNPV-PK2-EGFP infected cells evidently decreased from $48 \mathrm{~h}$ when Ac-PK2 protein was over expressed (Fig. 2a). In AcMNPV infected cells, phosphorylated eIF2 $\alpha$ was observed beginning at

a

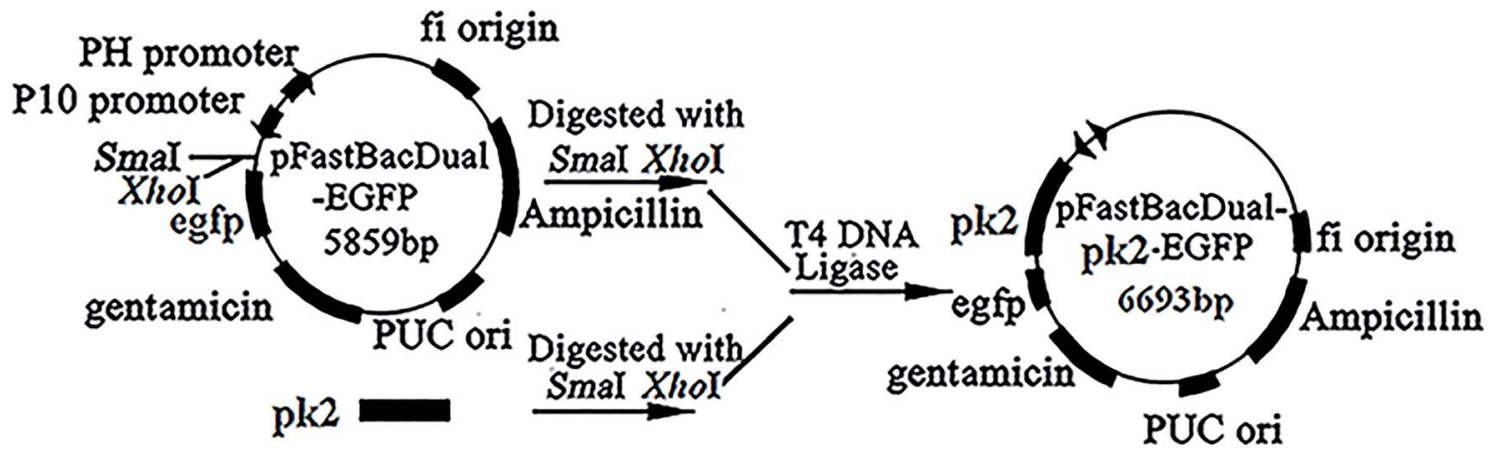

b

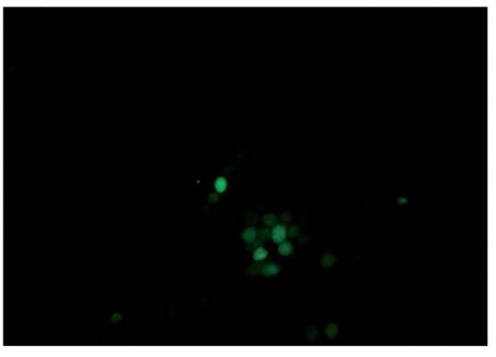

( I )

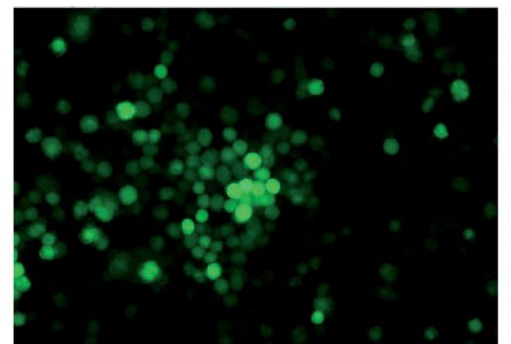

( II )

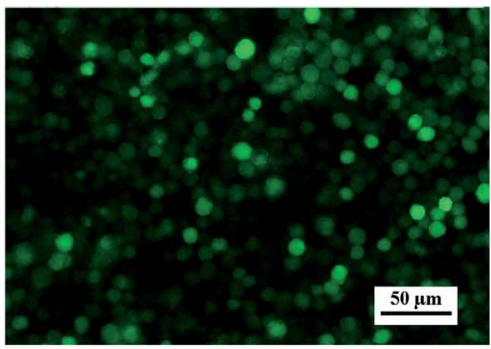

( III)

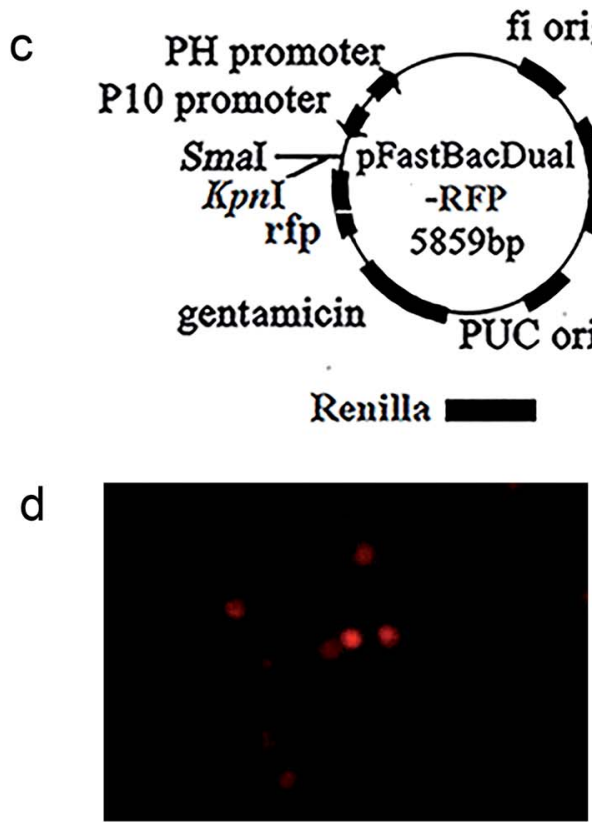

( I )

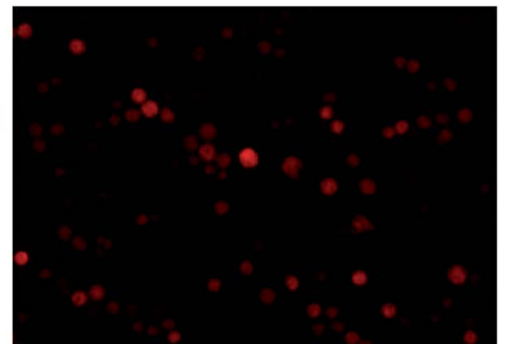

( II )
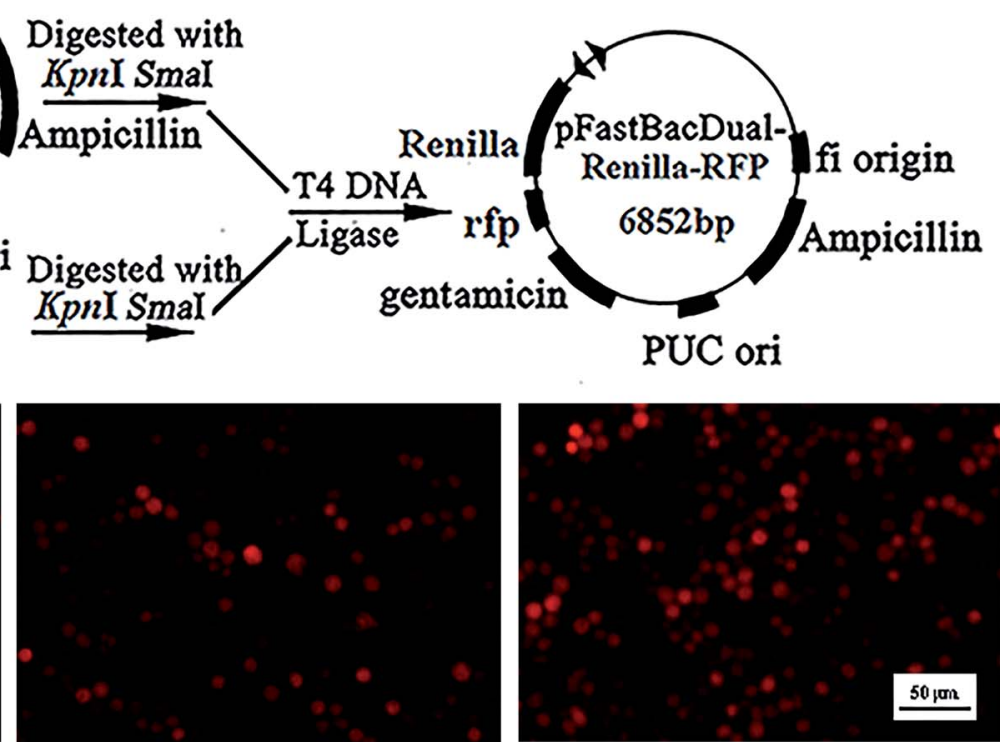

(III)

Fig. 1 Construction of AcMNPV-PK2-EGFP and AcMNPV-Renilla-RFP. (a) and (c) The physical and genetic map of pFastBauDual-pk2-egfp and pFastBauDual-renilla-rfp. (b) and (d) The (I), (II), (III) generation viruses of AcMNPV-PK2-EGFP and AcMNPV-Renilla-RFP were observed by fluorescence microscopy. 
a

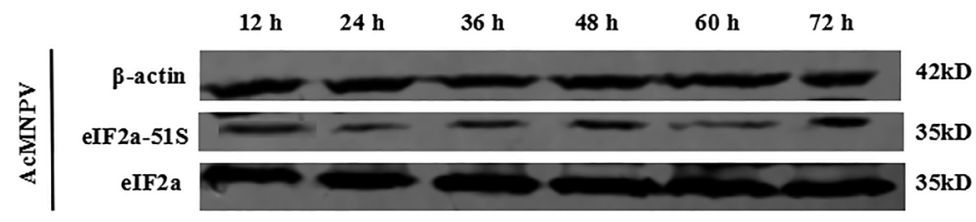

b

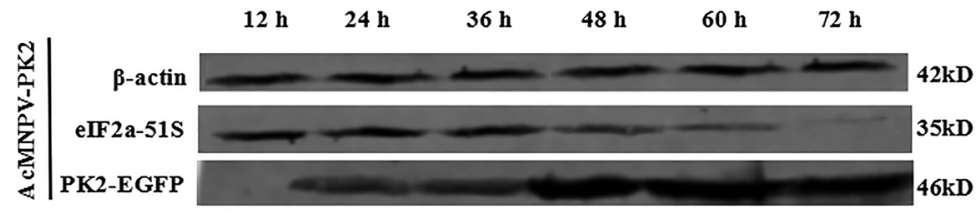

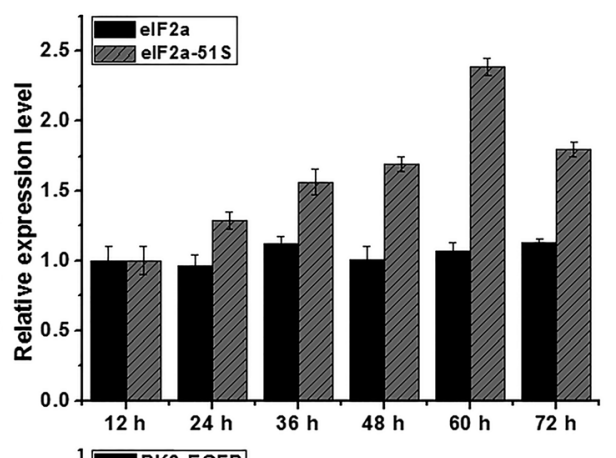

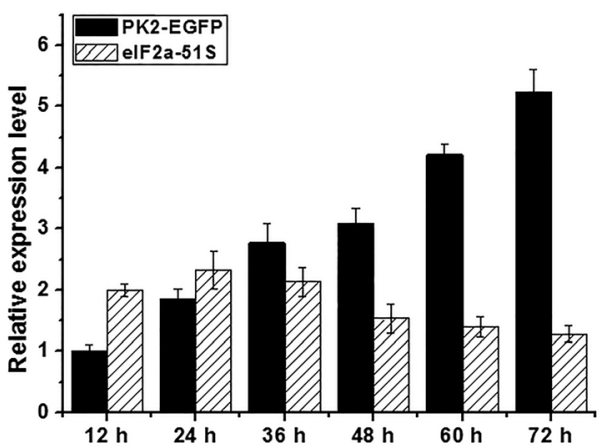

Fig. 2 AcMNPV-PK2-EGFP decreased elF2 $\alpha$ phosphorylation. (a) The expression of elF2 $\alpha$ and phosphorylation of elF2 $\alpha$ in AcMNPV group. (b) The expression of fusion protein PK2-EGFP and phosphorylation of elF2 $\alpha$ in AcMNPV-PK2-EGFP infected Sf9 cells. The right bars were grayscale scans of western blot lines.

$12 \mathrm{~h}$ p.i. and increased continuously until $60 \mathrm{~h}$ p.i. (Fig. $2 \mathrm{~b}$ ). The result suggested that PK2 protein overexpression could inhibit eIF2 $\alpha$ phosphorylation in Sf9 cells.

\section{AcMNPV-PK2-EGFP improved the total protein and foreign protein renilla luciferase synthesis}

Previous study indicated that viruses could markedly influence mRNA transcription and protein translation in host cells to help their replication. ${ }^{12}$ In order to explore the influence of Ac-PK2 protein on total protein translation in Sf9 cells, we measured the total protein content in Sf9 cells treated with AcMNPV or AcMNPV-PK2-EGFP. Fig. S2a $\uparrow$ showed that the total protein level in AcMNPV-PK2-EGFP treated cells increased by $13.61 \%$ at $48 \mathrm{~h}$ and $15.7 \%$ at $60 \mathrm{~h}$, compared with that in control group. To analyze the influence of Ac-PK2 on exogenous protein translation, we detected the renilla luciferase activity in AcMNPVRenilla-RFP-infected Sf9 cells, which were co-infected with AcMNPV or AcMNPV-PK2-EGFP, respectively. As shown in Fig. S2b, $\uparrow$ there were no obvious difference at the $24,36 \mathrm{~h}$ p.i., but at $48-72 \mathrm{~h}$ p.i., the renilla luciferase activity in AcMNPVPK2-EGFP treatment group significantly exceeded AcMNPVinfected Sf9 cells. These results indicated that Ac-PK2 protein overexpression would help exogenous protein and total protein synthesis, which would be helpful for the production of progeny virus.

\section{AcMNPV-PK2-EGFP effected the energy metabolism in host cell}

Intracellular ATP mainly comes from aerobic respiration, and glucose is the most important carbon source in culture medium of Sf9 cells. Fig. 3a showed the glucose consumption rate in Sf9 cells. Compared with control group, the glucose consumption rate in AcMNPV treatment group had an increase from $12 \mathrm{~h}$ p.i. to $60 \mathrm{~h}$ p.i. In AcMNPV-PK2-EGFP treatment group, glucose uptake had 1.16-fold, 1.22-fold and 1.34-fold increase at 36, 48, 60 h p.i., respectively, compared with the AcMNPV infected Sf9 cells. The accumulation of lactic acid in AcMNPV treatment group was 1.24 -fold, 1.30 -fold, 1.30 -fold and 1.36-fold higher than that in control group at $36,48,60,72 \mathrm{~h}$ p.i., respectively. Interestingly, the lactic acid accumulation had no obvious difference between AcMNPV-PK2-EGFP treatment group and control group (Fig. 3b). These results indicated that overexpression of Ac-PK2 protein could effect the glucose metabolism in infected Sf9 cells. We found that AcMNPV-PK2-EGFP could improve the total protein content and foreign protein renilla luciferase expression. These data suggested that overexpression of Ac-PK2 protein might effect not only the synthesis of protein, but also the energy metabolism pathway during the infection process.

The content of ATP in virus infected insect cells has already been described previously. ${ }^{13,14}$ Sf9 cells have high energy metabolism and ATP synthesis by TCA, in both normal and infected cells. ${ }^{14,15}$ To reveal the influence of baculovirus on the ATP content in Sf9 cells, control group, AcMNPV treatment group, AcMNPV-PK2-EGFP treatment group were compared. In Fig. 3c, the ATP content in AcMNPV treatment group significantly increased, which was consistent with previous research. The ATP content of AcMNPV-PK2 treatment group increased approximately $10 \%$, which was 1.12 -fold, 1.09 -fold, 1.10 -fold than those in AcMNPV infected Sf9 cells at 36, 48, $60 \mathrm{~h}$ p.i. The dynamical change of ATP/ADP in tissue cells is the basis of life 
a

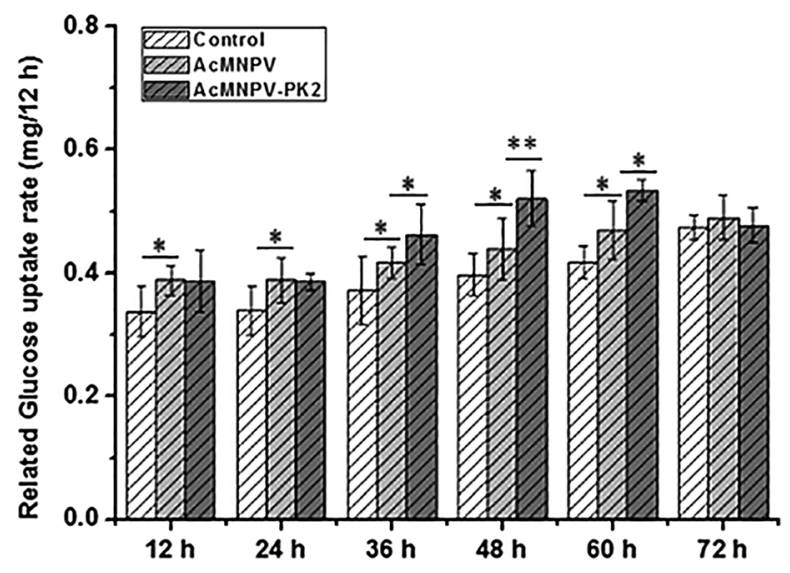

C

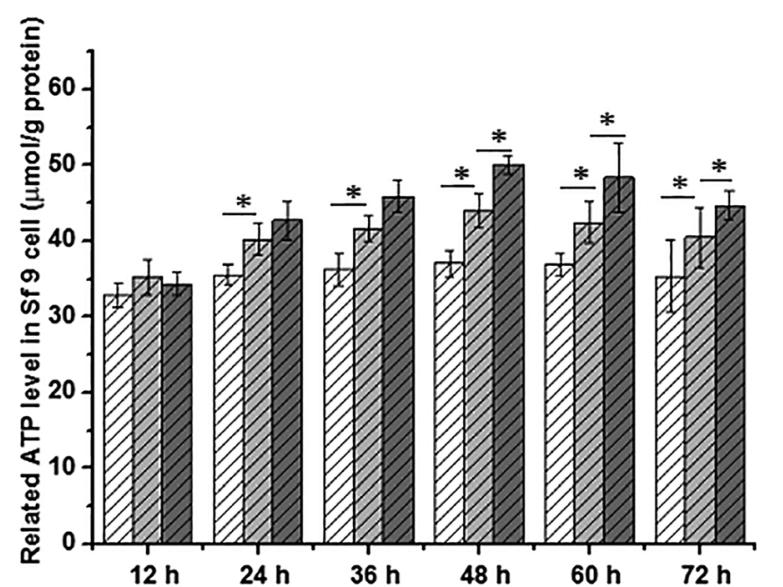

b

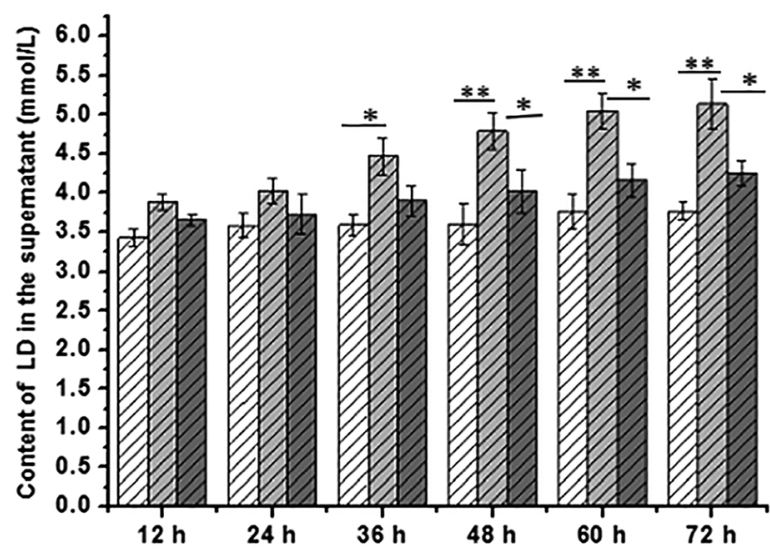

d

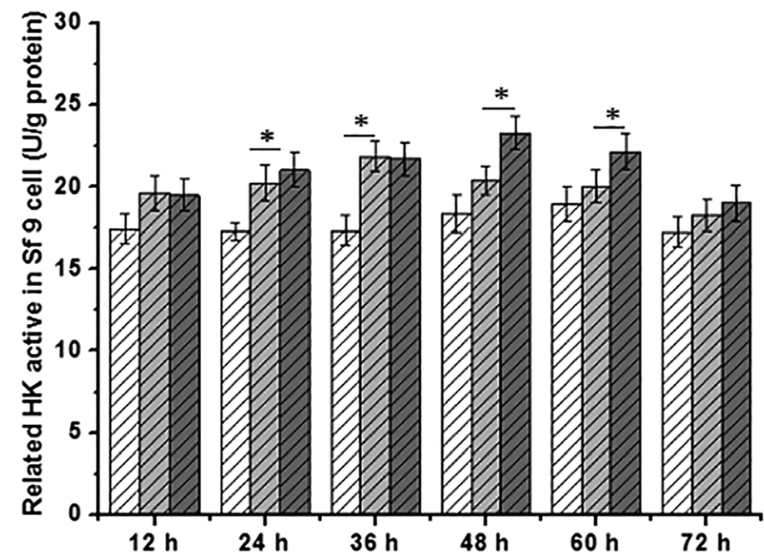

Fig. 3 Changes of energy metabolism in infected Sf9 cells. (a) Glucose consumption rate of Sf9 cells in AcMNPV and AcMNPV-PK2-EGFP treatment groups. (b) The lactic acid accumulation of supernatant in AcMNPV and AcMNPV-PK2-EGFP treatment groups. (c) The ATP content in Sf9 cells infected with AcMNPV and AcMNPV-PK2-EGFP. (d) The HK activity in Sf9 cells infected with AcMNPV and AcMNPV-PK2-EGFP. Statistical significance was determined using Student's $t$-test. $*, P<0.05 ; * *, P<0.01$.

energy supply. ${ }^{\mathbf{1 6}}$ Therefore, the content of ATP in cells will not fluctuate too much. About $10 \%$ up-regulation reflected the change of energy demand in Sf9 cells. We also examined the activity of hexokinase (HK) in infected Sf9 cells (Fig. 3d). We found that the activity of HK in AcMNPV-PK2-EGFP treatment group were significantly higher than AcMNPV infected Sf9 cells at 36, 48, $60 \mathrm{~h}$. Considering the change of glucose consumption rate and the lactic acid accumulation, ATP content and HK activity in AcMNPV-PK2-EGFP infected cells, we concluded that the AcMNPV mediated overexpression of Ac-PK2 could effect the energy metabolism in host cells.

\section{AcMNPV-PK2-EGFP improved budded virus (BV) production}

It has been analyzed that AcMNPV-PK2-EGFP improved the total protein content in host cell. Then, we examined BV production in infected Sf9 cells. As shown in Fig. 4, the BV production had no significant difference between two groups at $24 \mathrm{~h}$. But at 48

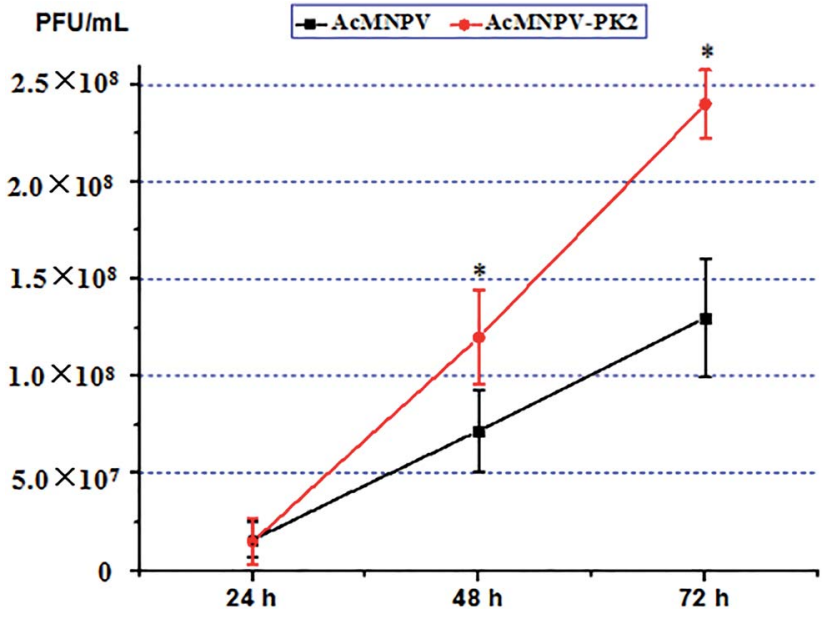

Fig. 4 Progeny virus production analysis of wild-type and AcMNPVPK2-EGFP in Sf9 cells. $* P<0.05$; Student's $t$-test. 
and $72 \mathrm{~h}$, BV production in AcMNPV-PK2-EGFP group remarkably exceeded wild type virus group.

\section{AcMNPV-PK2-EGFP promoted $S f 9$ cell apoptosis}

We detected the apoptosis of AcMNPV or AcMNPV-PK2-EGFP infected Sf9 cells by flow cytometry. Fig. 5a showed the EGFP fluorescence detection in AcMNPV-PK2-EGFP infected Sf9 cells. The apoptosis ratio of AcMNPV infected Sf9 cells was 9.97\%, 15.3\%, 21.5\%; while the apoptosis ratio of AcMNPV-PK2-EGFP infected Sf9 cells was $13.2 \%, 20.46 \%, 33.24 \%$, at 24, 48, $72 \mathrm{~h}$ (Fig. 5b). Fig. 5c showed the statistical of apoptosis rate of two treatment groups. At 48, $72 \mathrm{~h}$, apoptosis ratio of AcMNPV-PK2EGFP infected Sf9 cells significantly greater than that in AcMNPV treatment group. It's obvious that, compared with AcMNPV group, recombinant baculovirus AcMNPV-PK2-EGFP improved the apoptosis process of Sf9 cells.
AcMNPV-PK2-EGFP reduced the eIF2 $\alpha$ phosphorylation in the midgut of infected Spodoptera exigua larvae

Hao et al. found that the expressed BmK IT improved insecticidal efficiency of AcMNPV against Helicoverpa armigera larvae. ${ }^{17}$ Our result revealed that eIF2 $\alpha$ phosphorylation was decreased in AcMNPV-PK2-EGFP infected Sf9 cells. Then we detected the phosphorylation of eIF $2 \alpha$ in midgut of Spodoptera exigua larvae. As shown in Fig. S3, $\dagger$ phosphorylated eIF2 $\alpha$ progressively increased in the AcMNPV and AcMNPV-BmK IT treatment groups. But in the AcMNPV-PK2 and AcMNPV-PK2 + AcMNPV-BmK IT treatment groups, phosphorylated eIF2 $\alpha$ attained the maximum at 4 and $8 \mathrm{~h}$, respectively, then decreased. These results suggested that Ac-PK2 protein reduced eIF $2 \alpha$ phosphorylation in midgut tissue of AcMNPV-PK2 and AcMNPV-PK2 + AcMNPV-BmK IT infected beet armyworm, which might provide an advantageous environment for the

a
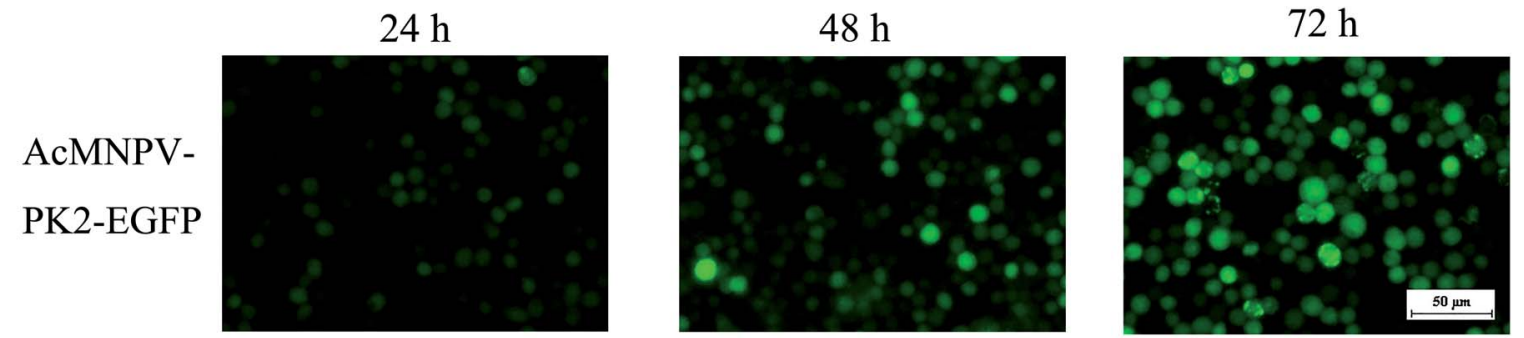

b
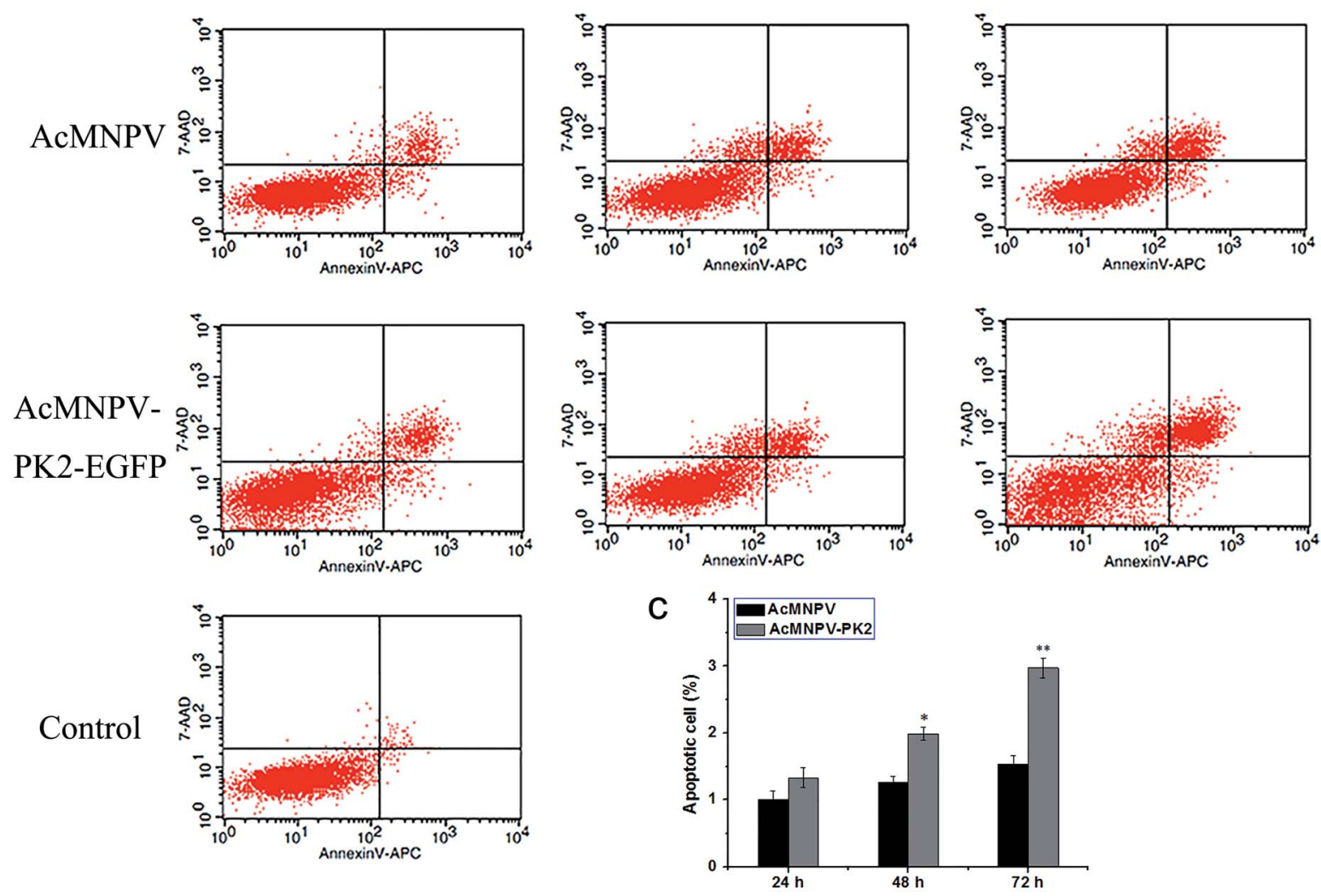

Fig. 5 Cell apoptosis rate analysis. (a) The fluorescence analysis of Sf9 cells infected with AcMNPV-PK2-EGFP. (b) Cell apoptosis rate of AcMNPV and AcMNPV-PK2-EGFP treatment group was detected by flow cytometric analysis. (c) The histogram of the cell apoptosis rate of AcMNPV or AcMNPV-PK2-EGFP treatment group. Statistical significance was determined using Student's $t$-test. $*, P<0.05 ; * *, P<0.01$. 

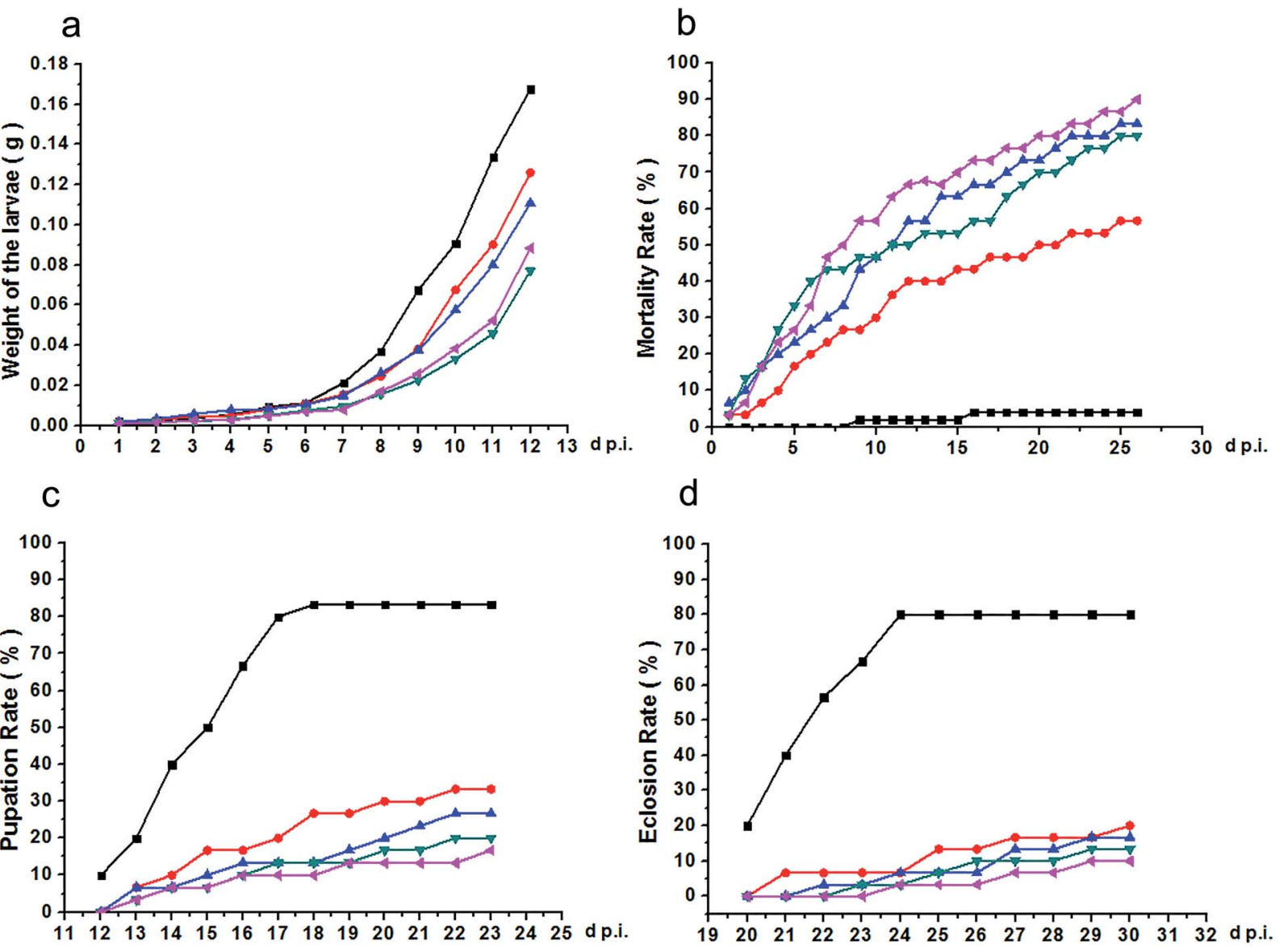

Fig. 6 Anti-insect activity analysis of AcMNPV, AcMNPV-PK2-EGFP, AcMNPV-BmK IT, and AcMNPV-PK2-EGFP + AcMNPV-BmK IT against the larvae of Spodostera exigua. Each treatment group contains 36 larvae. (a) The relationship between the weight of larvae and treatment time (d p.i.). (b) Mortality rate analysis. (c) Pupation rate analysis. (d) Eclosion rate analysis.

progeny virus replication, and be beneficial to improve the insect-resistant activity of AcMNPV.

\section{Co-infection of AcMNPV-PK2-EGFP and AcMNPV-BmK IT had high anti-insect activity}

Previously, we revealed that AcMNPV-PK2-EGFP could promote Sf9 cell apoptosis. Next, we analyzed the insecticidal efficiency of AcMNPV-PK2-EGFP. As shown in Fig. 6, we found that viruses infected larvae grown slowly than control, and had larger fatality rates and lower pupation rates and eclosion rates. Compared with AcMNPV, larvae growth speed in AcMNPV-PK2EGFP infected Spodoptera exigua was slow, only 0.83-fold than that of the former. The mortality rate in AcMNPV-BmK IT + AcMNPV-PK2-EGFP group was higher, which was 1.13-fold higher than AcMNPV-BmK IT group. Although AcMNPV-PK2 or AcMNPV-BmK IT had higher insecticidal activity compared with AcMNPV, the insecticidal activity of AcMNPV-PK2 + AcMNPV$B m \mathrm{~K}$ IT was more obvious than each of them. Combined with the previous experimental data, we concluded that AcMNPV mediated overexpression of Ac-PK2 protein could help AcMNPV$B m \mathrm{~K}$ IT replication, increase the efficiency of infection, and reduce insect growth rate.

\section{Discussion}

\section{AcMNPV-PK2-EGFP decreased eIF2 $\alpha$ phosphorylation and improved protein synthesis}

eIF2 is a hetero-trimeric ( $\alpha, \beta, \gamma$ subunits) GTPase, which delivers initiator methionyl-tRNA to the $40 \mathrm{~S}$ ribosome when translation initiation occurs. Under pressure situations, eIF2 $\alpha$ can be phosphorylated by eIF2 $\alpha$ kinases at a conserved site (Ser 51 in human eIF2 $\alpha$ ). PK2 protein exists specifically in a subset of alpha baculoviruses that are closely related to BmNPV and AcMNPV. ${ }^{4}$ It was possible that the Ac-pk2 gene was evolved from the host insect. Aarti et al. found that compared with wild-type virus, absence of $p k 2$ gene leaded to phosphorylated eIF $2 \alpha$ increase in infected cells. ${ }^{18}$ In this study, we constructed recombinant AcMNPV-PK2-EGFP, which could mediate the overexpression of Ac-PK2 in infected Sf9 cells. Our results 
revealed that Sf9 cells infected with AcMNPV-PK2-EGFP had lower level of phosphorylated eIF $2 \alpha$ than wild-type virus infection, which might provide a more favorable environment for virus replication. As a result, protein synthesis in AcMNPV-PK2EGFP infected Sf9 cells was up-regulated. So, we can try to improve the exogenous protein yield of insect baculovirus expression system by co-infected with AcMNPV-PK2-EGFP at least in this valid aspect.

\section{AcMNPV-PK2-EGFP effected the energy metabolism in host cells}

During the infection process, the generation of progeny viruses can be inhibited by the expression of host genes. The responses of host cell is different after infection and viruses can reprogramme some responses of host cell to increase the system productivity. ${ }^{19}$ Interestingly, we found that the glucose uptake increased and lactic acid accumulation decreased in AcMNPVPK2-EGFP infected Sf9 cells, compared with AcMNPV group. Previous studies indicated that virus infection had a significant influence on the energy metabolism of cells. The metabolic byproduct decreased and ATP synthesis upregulated in infected Sf9 cells. ${ }^{20} \mathrm{Sf} 9$ cells have high metabolic levels, so it may provide a more beneficial condition for baculovirus replication and progeny virus maturation. ${ }^{7}$ We further detected the ATP content and HK activity in virus-infected Sf9 cells. Results showed that ATP content and the activity of HK were effected in AcMNPV-
PK2-EGFP infected Sf9 cells. We concluded that Ac-PK2 overexpression re-directed metabolism fluxome of glucose to create a better environment for protein translation and DNA replication. Fig. 7 showed the proposed mechanism of AcMNPV-PK2EGFP to improve the protein synthesis and alter the metabolism of host cells.

\section{Overexpression of Ac-PK2 protein was beneficial to progeny virions formation, and promoted apoptosis of host cells}

Previous studies revealed that, compared with cells infected with AcMNPV virus, KO-infected BmN cells had higher expression levels of phospho-eIF2 $\alpha$ and lower levels of BV production. ${ }^{4}$ Our results demonstrated that BV production was increased, and eIF2 $\alpha$ phosphorylation was reduced in AcMNPV-PK2-EGFP infected Sf9 cells, compared with AcMNPV treatment group. It is known that cell apoptosis can be induced by external stimulus and internal factors. ${ }^{21}$ In virus-infected cells, apoptosis is an important defence mechanism in normal development. ${ }^{22,23}$ The infection of baculovirus can lead to changes in cell morphology and metabolism, which may induce apoptosis in the host cells. Flow cytometry analysis showed that the apoptosis of Sf9 cells infected by AcMNPV-PK2-EGFP significantly increased at 48 and $72 \mathrm{~h}$, compared with AcMNPV. In summary, our findings raised the intriguing possibility that AcMNPV-PK2-EGFP had higher cytotoxicity because it could improve the progeny virions formation, which in turn triggered the apoptosis of host cells.

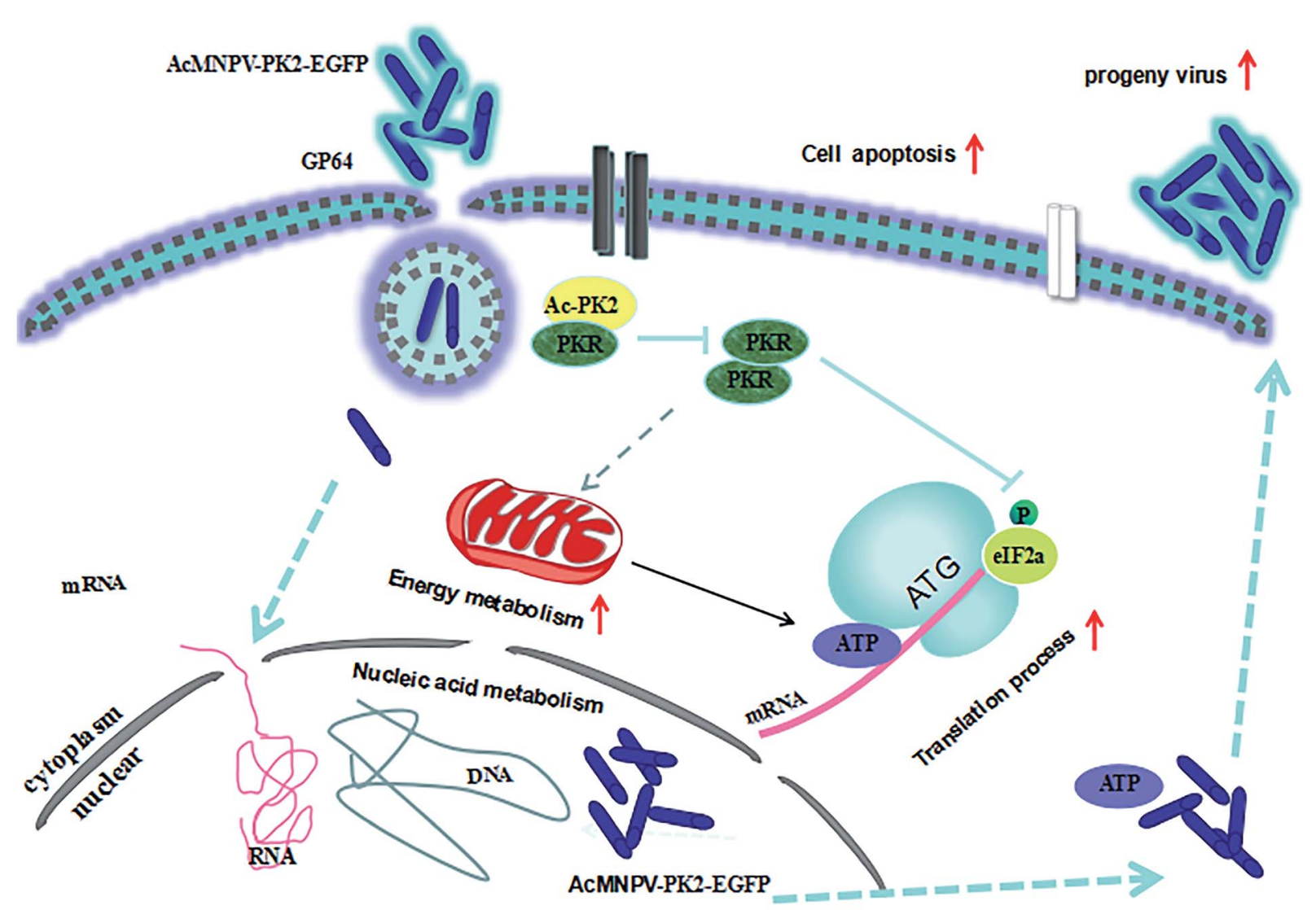

Fig. 7 Proposed mechanism model of AcMNPV-PK2-EGFP improved the protein synthesis and re-directed the metabolism of host cell. 
Co-infection of AcMNPV-PK2-EGFP and AcMNPV-BmK IT improved insect-resistant activity against the larva of Spodoptera exigua

In order to improve the virulence of baculovirus-based biopesticides, we can insert some insect toxins gene into AcMNPV genome to improve its insecticidal activity. ${ }^{24}$ Our previous work revealed that AcMNPV-BmK IT enhanced the insecticidal activity of AcMNPV against Helicoverpa armigera larvae. ${ }^{9}$ In this study, western blot analysis showed that AcMNPV-PK2-EGFP could inhibit host eIF2 $\alpha$ phosphorylation in both cells and insects (Spodoptera exigua). Thus, Ac-PK2 protein could not only inhibit eIF $2 \alpha$ phosphorylation in host cell, but also in insects. Insect experiment results demonstrated that the co-infection of recombinant baculovirus AcMNPV-PK2-EGFP and AcMNPV$B m \mathrm{~K}$ IT enhanced the insect-resistant activity of baculovirus. We inferred this was due to the facilitating effect of AcMNPV-PK2EGFP on the expression of BmK IT. The high toxicity and strong host-specificity of co-infection by recombinant baculovirus prompted it to be a new potential biological insecticide, but the detailed regulatory mechanism of the recombinant baculovirus remained to be explored.

Since the 1970 s, scholars made safety testing of wild baculovirus for all kinds of creatures and even people, almost all tests proved that it was safe to use wild baculovirus as insecticide. ${ }^{25}$ The bio-safety of recombinant baculovirus insecticides had raised concerns because they added exogenous genes or removed certain genes, such as $B m \mathrm{~K}$ IT gene. This was the reason why recombinant baculovirus bio-insecticides were rarely approved for environmental release, commercial production and application. The recombinant baculovirus insecticides were still in the stage of laboratory research and small area field tests. Although the $p k 2$ gene in recombinant virus AcMNPV-PK2-EGFP is AcMNPV's own gene, it is also necessary to be further verified by a large number of field applications and environmental release tests. We believe that recombinant baculovirus insecticides will better serve the field of plant protection, along with the comprehensive and in-depth development of basic research on the safety of engineered recombinant baculoviruses.

\section{Conclusion}

In summary, we constructed a recombinant AcMNPV-PK2-EGFP virus in this study, and we found it could help the expression of foreign proteins in infected cells by rescuing the translation initial. So it might be used to improve the production efficiency of insect baculovirus expression system in the future. We also found that the recombinant AcMNPV-PK2-EGFP virus had higher insecticidal activity than wild virus, and it improved the insecticidal activity of recombinant virus AcMNPV-BmK IT, so it could be used as a potential biological pesticides or auxiliary insecticide. At present, insect baculovirus insecticides have been applied in many countries, ${ }^{26}$ but how to develop the advantages of recombinant insect baculovirus insecticides, and overcome the disadvantages to make them more widely used are also the research direction in the future. To avoid potential threats to natural environment, human and animals, safety evaluation of recombinant insect baculovirus insecticides should be conducted continuously.

\section{Compliance with ethical standards}

All applicable international, national, and/or institutional guidelines for the care and use of animals were followed.

\section{Conflicts of interest}

The authors declare that they have no conflict of interest.

\section{Acknowledgements}

The present work was supported by grants from National Natural Science Foundation of China [No. 31272100 and 31372199] to Y. Fu and A. Liang, respectively. And it was also supported by grants from Natural Science Foundation of Shanxi Province [No. 2014011038-1] and Shanxi '1331 Project' Collaborative Innovation Center (1331 CIC) to Y. Fu.

\section{References}

1 M. D. Ayres, S. C. Howard, J. Kuzio, M. Lopez-Ferber and R. D. Possee, Virology, 1994, 202, 586-605.

2 Y. Li and L. K. Miller, Virology, 1995, 206, 314-323.

3 T. E. Dever, R. Sripriya, J. R. McLachlin, J. Lu, J. R. Fabian, S. R. Kimball and L. K. Miller, Proc. Natl. Acad. Sci. U. S. A., 1998, 95, 4164-4169.

4 J. J. Li, C. Cao, S. M. Fixsen, J. M. Young, C. Ono, H. Bando, N. C. Elde, S. Katsuma, T. E. Dever and F. Sicheri, Proc. Natl. Acad. Sci. U. S. A., 2015, 112, E4364-E4373.

5 V. Bernal, N. Carinhas, A. Y. Yokomizo, M. J. T. Carrondo and P. M. Alves, Biotechnol. Bioeng., 2009, 104, 162-180.

6 V. Bernal, F. Monteiro, N. Carinhas, R. Ambrosio and P. M. Alves, J. Biotechnol., 2010, 150, 332-342.

7 F. Monteiro, V. Bernal and P. M. Alves, Biotechnol. Bioeng., 2017, 114, 674-684.

8 J. K. Moulton, D. A. Pepper, R. K. Jansson and T. J. Dennehy, J. Econ. Entomol., 2002, 95, 414-424.

9 X. J. Fan, B. Zheng, Y. J. Fu, Y. Sun and A. H. Liang, Chin. Sci. Bull., 2008, 53, 1855-1860.

10 S. Katsuma, Y. Koyano, W. Kang, R. Kokusho, S. G. Kamita and T. Shimada, PLoS Pathog., 2012, 8, e1002644.

11 C. W. Wu and S. Wang, J. Virol., 2012, 86, 484-491.

12 D. Vester, E. Rapp, D. Gade, Y. Genzel and U. Reichl, Proteomics, 2009, 9, 3316-3327.

13 P. E. Cruz, P. C. Martins, P. M. Alves, C. C. Peixoto, H. Santos, J. L. Moreira and M. J. T. Carrondo, Biotechnol. Bioeng., 1999, 65, 133-143.

14 A. M. Olejnik, K. Czaczyk, R. Marecik, W. Grajek and T. Jankowski, Appl. Microbiol. Biotechnol., 2004, 65, 18-24.

15 J. Neermann and R. Wagner, J. Cell. Physiol., 1996, 166, 152169.

16 B. Korzeniewski, Biochem. J., 1998, 330, 1189-1195. 
17 C. J. Hao, C. G. Xu, W. Wang, B. F. Chai and A. H. Liang, Biotechnol. Lett., 2005, 27, 1929-1934.

18 I. Aarti, K. Rajesh and K. V. Ramaiah, Apoptosis, 2010, 15, 679-692.

19 S. M. Thiem, In Vitro Cell. Dev. Biol.: Anim., 2009, 45, 111126.

20 J. Ritter, A. Wahl, S. Freund, Y. Genzel and U. Reichl, BMC Syst. Biol., 2010, 4, 61.

21 R. S. Sloviter, Trends Pharmacol. Sci., 2002, 23, 19-24.
22 A. Bergmann, J. Agapite and H. Steller, Oncogene, 1998, 17, 3215-3223.

23 E. H. Baehrecke, Nat. Rev. Mol. Cell Biol., 2002, 3, 779-787.

24 M. B. Ashour, D. A. Ragheb, E. S. A. El-Sheikh, E. A. A. Gomaa, S. G. Kamita and B. D. Hammock, Int. J. Environ. Res. Public Health, 2007, 4, 111-125.

25 F. Moscardi, Annu. Rev. Entomol., 1999, 44, 257-289.

26 C. R. Smith, K. M. Heinz, C. G. Sansone and J. L. Flexner, J. Econ. Entomol., 2000, 93, 1109-1117. 